\title{
Optimality Conditions and Duality for Multiobjective Variational Problems with Generalized $\rho-(\boldsymbol{\eta}, \boldsymbol{\theta})$-B-Type-I Functions
}

\author{
C. Nahak ${ }^{1}$ and N. Behera ${ }^{1,2}$ \\ ${ }^{1}$ Department of Mathematics, Indian Institute of Technology Kharagpur, Kharagpur-721302, India \\ ${ }^{2}$ Department of Mathematics, Applied Sciences and Humanities, ITS Engineering College Greater Noida, UP, India \\ Correspondence should be addressed to C. Nahak, cnahak@maths.iitkgp.ernet.in
}

Received 24 June 2010; Revised 13 December 2010; Accepted 15 March 2011

Academic Editor: Derong Liu

Copyright () 2011 C. Nahak and N. Behera. This is an open access article distributed under the Creative Commons Attribution License, which permits unrestricted use, distribution, and reproduction in any medium, provided the original work is properly cited.

We use $\rho-(\eta, \theta)$ - $B$-type-I and generalized $\rho-(\eta, \theta)$ - $B$-type-I functions to establish sufficient optimality conditions and duality results for multiobjective variational problems. Some of the related problems are also discussed.

\section{Introduction}

The relationship between mathematical programming and classical calculus of variation was explored and extended by Hanson [1]. Thereafter variational programming problems have some attention in the literature. Mond and Hanson [2] obtained optimality conditions and duality results for scalar valued variational problems under convexity assumptions. Motivated by the approach by Bector and Husain [3], Nahak and Nanda [4] and later Bhatia and Mehra [5] extended the results of Mond et al. [6] to multiobjective variational problems involving invex functions and generalized $B$-invex functions, respectively. Analogous results were developed by Zalmai [7] for fractional variational programming problem containing arbitrary norms and by Liu [8] for generalized fractional case involving $(F, \rho)$-convex functions.

Type-I functions were first introduced by Hanson and Mond [9], and Rueda and Hanson [10] defined a class of pseudo-type-I and quasi-type-I functions as generalization of type-I functions. Bhatia and Mehra [5] studied the optimality conditions and duality results for multiobjective variational problems involving generalized $B$-invexity. We use $\rho-(\eta, \theta)$ - $B$-type-I and generalized $\rho-(\eta, \theta)$ - $B$-typeI functions to establish sufficient optimality conditions and duality results for multiobjective variational problems.
In Section 2 of this paper, we introduce $\rho-(\eta, \theta)-B$ type-I and generalized $\rho-(\eta, \theta)$ - $B$-type-I functions for the continuous case. Using these new classes of functions, we establish various sufficient optimality conditions in Section 3 of this paper. Section 4 is devoted to the duality results. Some related problems are discussed in Section 5. The results obtained in this paper are more general than those obtained in $[4,5,11]$.

Let $I=[a, b]$ be a real interval, let $f: I \times \mathbb{R}^{n} \times \mathbb{R}^{n} \rightarrow$ $\mathbb{R}^{p}$, and let $h: I \times \mathbb{R}^{n} \times \mathbb{R}^{n} \rightarrow \mathbb{R}^{m}$ be continuously differentiable functions with respect to their arguments and $x: I \rightarrow \mathbb{R}^{n}, \dot{x}$ denotes the derivative of $x$ with respect $t$. Denote the partial derivative of scalar valued function $g$ : $I \times \mathbb{R}^{n} \times \mathbb{R}^{n} \rightarrow \mathbb{R}$, with respect to $t, x$, and $\dot{x}$, respectively, by $g_{t}, g_{x}$, and $g_{\dot{x}}$ such that $g_{x}=\left[\partial g / \partial x_{1}, \ldots, \partial g / \partial x_{n}\right], g_{\dot{x}}=$ $\left[\partial g / \partial \dot{x}_{1}, \ldots, \partial g / \partial \dot{x}_{n}\right]$. Similarly we write the partial derivative of the vector functions $f$ and $h$ using matrices with $p$ and $m$ rows, respectively, instead of one. Let $C\left(I, \mathbb{R}^{n}\right)$ denote the space of piecewise smooth functions $x$ with norm $\|x\|=$ $\|x\|_{\infty}+\|D x\|_{\infty}$, where the differential operator $D$ is given by

$$
u=D x \Longleftrightarrow x(t)=x(u)+\int_{a}^{t} u(s) d s
$$

Therefore, $D=d / d t$ except at discontinuities. 
Consider the following multiobjective variational problem:

$$
\begin{aligned}
(V P) \text { minimize } & \int_{a}^{b} f(t, x, \dot{x}) d t \\
& =\left(\int_{a}^{b} f_{1}(t, x, \dot{x}), \ldots, \int_{a}^{b} f_{p}(t, x, \dot{x})\right) \\
\text { subject to } & x(a)=\alpha, \quad x(b)=\beta, \\
& h(t, x, \dot{x}) \leqq 0, \quad t \in I .
\end{aligned}
$$

Let $K=\left\{x \in C\left(I, \mathbb{R}^{n}\right) \mid x(a)=\alpha, x(b)=\beta, h(t, x, \dot{x}) \leqq\right.$ $0, t \in I\}$.

Efficiency and proper efficiency are defined in their usual sense as defined in [4].

In relation to $(V P)$, we introduce the following multiobjective problem $\left(P_{k}^{*}\right)$, for each $k=1, \ldots, p$, each problem with single objective:

$$
\begin{array}{rl}
\left(P_{k}^{*}\right) \text { Minimize } & \int_{a}^{b} f_{k}(t, x, \dot{x}) d t \\
\text { Subject to } \quad & x(a)=\alpha, \quad x(b)=\beta, \\
& \int_{a}^{b} f_{i}(t, x, \dot{x}) d t \leqq \int_{a}^{b} f_{i}(t, * * * * *) \\
x & i=1,2, \ldots, p, \quad i \neq k, \\
& h_{j}(t, x, \dot{x}) \leqq 0, \quad j=1, \ldots, m, t \in I .
\end{array}
$$

The following lemma can be established on the lines of Chankong and Haimes [12].

Definition 1 (see [4]). A point $\stackrel{*}{x}$ in $K$ is said to be an efficient solution of $(V P)$ if for all $x \in K$

$$
\begin{aligned}
& \int_{a}^{b} f_{i}(t, \stackrel{*}{x}, \stackrel{*}{x}) d t \geq \int_{a}^{b} f_{i}(t, x, \dot{x}) d t, \quad \forall i \in\{1, \ldots, p\} \\
& \Longrightarrow \int_{a}^{b} f_{i}(t, \stackrel{*}{x}, \stackrel{*}{x}) d t=\int_{a}^{b} f_{i}(t, x, \dot{x}) d t, \quad \forall i \in\{1, \ldots, p\} .
\end{aligned}
$$

Definition 2 (see [13]). A point $\stackrel{*}{x}$ in $K$ is said to be a properly efficient solution of (VP) if for all $x \in K$ there exists a scalar $M>0$ such that for all $i \in\{1, \ldots, p\}$

$$
\begin{gathered}
\int_{a}^{b} f_{i}(t, \stackrel{*}{x}, \dot{*} x) d t-\int_{a}^{b} f_{i}(t, x, \dot{x}) d t, \quad \forall i \in\{1, \ldots, p\} \\
\leq M\left(\int_{a}^{b} f_{j}(t, x, \dot{x}) d t-\int_{a}^{b} f_{j}(t, \stackrel{*}{x}, \dot{*} x) d t\right)
\end{gathered}
$$

for some $j$ such that

$$
\int_{a}^{b} f_{j}(t, x, \dot{x}) d t>\int_{a}^{b} f_{j}(t, \stackrel{*}{x}, \stackrel{*}{x}) d t
$$

whenever $x$ is in $K$ and

$$
\int_{a}^{b} f_{j}(t, x, \dot{x}) d t<\int_{a}^{b} f_{j}(t, \stackrel{*}{x}, \stackrel{*}{x}) d t .
$$

Lemma 3. $\stackrel{*}{x}$ is an efficient solution of $(V P)$ if and only if ${ }^{*}$ is an optimal solution of $\left(P_{k}^{*}\right)$ for each $k=1,2, \ldots, p$.

Theorem 4 (see [2]). For every optimal normal solution of $\left(P_{k}^{*}\right)$, for each $k=1,2, \ldots, p$, there exist real numbers $\lambda_{1 k}, \ldots, \lambda_{p k}$ with $\lambda_{k k}=1$ and piecewise smooth function $y_{k}$ : $I \rightarrow \mathbb{R}^{m}$ such that

$$
\begin{gathered}
f_{k x}(t, \stackrel{*}{x}, \stackrel{*}{x})+\sum_{\substack{i=1 \\
i \neq k}}^{p} \lambda_{i k} f_{i x}(t, \stackrel{*}{x}, \underset{*}{x})+y_{k}(t)^{T} h_{x}(t, \stackrel{*}{x}, \stackrel{*}{x}) \\
=\frac{d}{d t}\left(f_{k \dot{x}}(t, \stackrel{*}{x}, \underset{*}{x})+\sum_{\substack{i=1 \\
i \neq k}}^{p} \lambda_{i k} f_{i x}(t, \stackrel{*}{x}, \stackrel{*}{x})+y_{k}(t)^{T} h_{\dot{x}}(t, \stackrel{*}{x}, \stackrel{*}{x})\right), \\
y_{k}(t)^{T} h(t, \underset{*}{*}, \underset{*}{x})=0, \quad t \in I, \\
y_{k}(t) \geqq 0, \quad t \in I, \\
\lambda_{i k} \geqq 0, \quad i=1,2, \ldots, p, \quad i \neq k .
\end{gathered}
$$

\section{2. $\rho-(\eta, \theta)-B$-Type-I and Generalized $\rho-(\eta, \theta)-B$-Type-I Functions}

Definition 5. Let $f$ and $h$ be real valued functions. A pair $(f, h)$ is said to be $\left(\rho_{0}, \rho_{1}\right)-(\eta, \theta)$-B-type-I at $u \in$ $C\left(I, \mathbb{R}^{n}\right)$ with respect to $b_{0}, b_{1}, \eta$, and $\theta$ if there exist functions $b_{0}, b_{1}: C\left(I, \mathbb{R}^{n}\right) \times C\left(I, \mathbb{R}^{n}\right) \rightarrow \mathbb{R}_{+}$and $\eta, \theta: I \times \mathbb{R}^{n} \times \mathbb{R}^{n} \rightarrow \mathbb{R}^{n}$ with $\eta(t, x, x)=0, \rho_{0}, \rho_{1} \in \mathbb{R}$ such that for all $x \in K$

$$
\begin{gathered}
b_{0}(x, u)\left\{\int_{a}^{b} f(t, x, \dot{x}) d t-\int_{a}^{b} f(t, u, \dot{u}) d t\right\} \\
\geqq \int_{a}^{b}\left[\eta(t, x, u)^{T} f_{x}(t, u, \dot{u})+\frac{d}{d t} \eta(t, x, u)^{T} f_{\dot{x}}(t, u, \dot{u})\right. \\
\left.+\rho_{0}\|\theta(t, x, u)\|^{2}\right] d t
\end{gathered}
$$

$$
\begin{aligned}
& -b_{1}(x, u) \int_{a}^{b} h(t, u, \dot{u}) d t \\
& \geqq \int_{a}^{b}\left[\eta(t, x, u)^{T} h_{x}(t, u, \dot{u})+\frac{d}{d t} \eta(t, x, u)^{T} h_{\dot{x}}(t, u, \dot{u})\right. \\
& \left.+\rho_{1}\|\theta(t, x, u)\|^{2}\right] d t .
\end{aligned}
$$

If, in the previous definition, (9) is satisfied as a strict inequality, then we say that a pair $(f, h)$ is $\left(\rho_{0}, \rho_{1}\right)-(\eta, \theta)-B$ semistrictly-type-I at $u \in C\left(I, \mathbb{R}^{n}\right)$ with respect to $b_{0}, b_{1}, \eta$, and $\theta$ and $\rho_{0}, \rho_{1} \in \mathbb{R}$.

Definition 6. A pair $(f, h)$ is said to be $\left(\rho_{0}, \rho_{1}\right)-(\eta, \theta)-B$ quasi-type-I at $u \in C\left(I, \mathbb{R}^{n}\right)$ with respect to $b_{0}, b_{1}, \eta, \theta$ if there exist functions $b_{0}, b_{1}: C\left(I, \mathbb{R}^{n}\right) \times C\left(I, \mathbb{R}^{n}\right) \rightarrow \mathbb{R}_{+}$and 
$\eta, \theta: I \times \mathbb{R}^{n} \times \mathbb{R}^{n} \rightarrow \mathbb{R}^{n}$ with $\eta(t, x, x)=0, \rho_{0}, \rho_{1} \in \mathbb{R}$ such that for all $x \in K$

$$
\begin{gathered}
b_{0}(x, u)\left\{\int_{a}^{b} f(t, x, \dot{x}) d t-\int_{a}^{b} f(t, u, \dot{u}) d t\right\} \leqq 0 \\
\Longrightarrow \int_{a}^{b}\left[\eta(t, x, u)^{T} f_{x}(t, u, \dot{u})+\frac{d}{d t} \eta(t, x, u)^{T} f_{\dot{x}}(t, u, \dot{u})\right. \\
\left.\quad+\rho_{0}\|\theta(t, x, u)\|^{2}\right] d t \leqq 0, \\
-b_{1}(x, u) \int_{a}^{b} h(t, u, \dot{u}) d t \leqq 0 \\
\Longrightarrow \int_{a}^{b}\left[\eta(t, x, u)^{T} h_{x}(t, u, \dot{u})+\frac{d}{d t} \eta(t, x, u)^{T} h_{\dot{x}}(t, u, \dot{u})\right. \\
\left.+\rho_{1}\|\theta(\mathrm{t}, x, u)\|^{2}\right] d t \leqq 0 .
\end{gathered}
$$

Definition 7. A pair $(f, h)$ is said to be $\left(\rho_{0}, \rho_{1}\right)-(\eta, \theta)-B$ strongly pseudo-type-I at $u \in C\left(I, \mathbb{R}^{n}\right)$ with respect to $b_{0}, b_{1}$, $\eta$, and $\theta$ if there exist functions $b_{0}, b_{1}: C\left(I, \mathbb{R}^{n}\right) \times C\left(I, \mathbb{R}^{n}\right) \rightarrow$ $\mathbb{R}_{+}$and $\eta, \theta: I \times \mathbb{R}^{n} \times \mathbb{R}^{n} \rightarrow \mathbb{R}^{n}$ with $\eta(t, x, x)=0, \rho_{0}, \rho_{1} \in \mathbb{R}$ such that for all $x \in K$,

$$
\begin{gathered}
\int_{a}^{b}\left[\eta(t, x, u)^{T} f_{x}(t, u, \dot{u})+\frac{d}{d t} \eta(t, x, u)^{T} f_{\dot{x}}(t, u, \dot{u})\right. \\
\left.+\rho_{0}\|\theta(t, x, u)\|^{2}\right] d t \geqq 0 \\
\Longrightarrow b_{0}(x, u)\left\{\int_{a}^{b} f(t, x, \dot{x}) d t-\int_{a}^{b} f(t, u, \dot{u}) d t\right\} \geqq 0, \\
\int_{a}^{b}\left[\eta(t, x, u)^{T} h_{x}(t, u, \dot{u})+\frac{d}{d t} \eta(t, x, u)^{T} h_{\dot{x}}(t, u, \dot{u})\right. \\
\left.+\rho_{1}\|\theta(t, x, u)\|^{2}\right] d t \geqq 0 \\
\Longrightarrow-b_{1}(x, u) \int_{a}^{b} h(t, u, \dot{u}) d t \geqq 0 .
\end{gathered}
$$

Clearly the class of $\left(\rho_{0}, \rho_{1}\right)-(\eta, \theta)$-B-quasi-type-I functions and the class of $\left(\rho_{0}, \rho_{1}\right)-(\eta, \theta)$-B-strongly pseudo-type-I functions are more general than the class of $\left(\rho_{0}, \rho_{1}\right)-(\eta, \theta)-$ $B$-type-I functions.

Definition 8. A pair $(f, h)$ is said to be $B-\rho_{0}-(\eta, \theta)$-quasi$\rho_{1}-(\eta, \theta)$-pseudo-type-I at $u \in C\left(I, \mathbb{R}^{n}\right)$ with respect to $b_{0}, b_{1}, \eta$, and $\theta$ if there exist functions $b_{0}, b_{1}: C\left(I, \mathbb{R}^{n}\right) \times$ $C\left(I, \mathbb{R}^{n}\right) \rightarrow \mathbb{R}_{+}$and $\eta, \theta: I \times \mathbb{R}^{n} \times \mathbb{R}^{n} \rightarrow \mathbb{R}^{n}$ with $\eta(t, x, x)=0, \rho_{0}, \rho_{1} \in \mathbb{R}$ such that for all $x \in K$

$$
\begin{gathered}
b_{0}(x, u)\left\{\int_{a}^{b} f(t, x, \dot{x}) d t-\int_{a}^{b} f(t, u, \dot{u}) d t\right\} \leqq 0 \\
\Longrightarrow \int_{a}^{b}\left[\eta(t, x, u)^{\mathrm{T}} f_{x}(t, u, \dot{u})+\frac{d}{d t} \eta(t, x, u)^{T} f_{\dot{x}}(t, u, \dot{u})\right. \\
\left.\quad+\rho_{0}\|\theta(t, x, u)\|^{2}\right] d t \leqq 0,
\end{gathered}
$$

$$
\begin{gathered}
\int_{a}^{b}\left[\eta(t, x, u)^{T} h_{x}(t, u, \dot{u})+\frac{d}{d t} \eta(t, x, u)^{T} h_{\dot{x}}(t, u, \dot{u})\right. \\
\left.+\rho_{1}\|\theta(t, x, u)\|^{2}\right] d t \geqq 0 \\
\Longrightarrow-b_{1}(x, u) \int_{a}^{b} h(t, u, \dot{u}) d t \geqq 0 .
\end{gathered}
$$

If, in the above definition, inequality (14) is satisfied as

$$
\begin{gathered}
\int_{a}^{b}\left[\eta(t, x, u)^{T} h_{x}(t, u, \dot{u})+\frac{d}{d t} \eta(t, x, u)^{T} h_{\dot{x}}(t, u, \dot{u})\right. \\
\left.+\rho_{1}\|\theta(t, x, u)\|^{2}\right] d t \geqq 0 \\
\Longrightarrow-b_{1}(x, u) \int_{a}^{b} h(t, u, \dot{u}) d t>0,
\end{gathered}
$$

then we say that a pair $(f, h)$ is $B-\rho_{0}-(\eta, \theta)$-quasi- $\rho_{1}-(\eta, \theta)$ strictly pseudo-type-I at $u \in C\left(I, \mathbb{R}^{n}\right)$ with respect to $b_{0}, b_{1}$, $\eta$, and $\theta$ and $\rho_{0}, \rho_{1} \in \mathbb{R}$.

Definition 9. A pair $(f, h)$ is said to be $B-\rho_{0}-(\eta, \theta)$ strongly pseudo- $\rho_{1}-(\eta, \theta)$-quasi-type-I at $u \in C\left(I, \mathbb{R}^{n}\right)$ with respect to $b_{0}, b_{1}, \eta, \theta$ if there exist functions $b_{0}, b_{1}$ : $C\left(I, \mathbb{R}^{n}\right) \times C\left(I, \mathbb{R}^{n}\right) \rightarrow \mathbb{R}_{+}$and $\eta, \theta: I \times \mathbb{R}^{n} \times \mathbb{R}^{n} \rightarrow \mathbb{R}^{n}$ with $\eta(t, x, x)=0, \rho_{0}, \rho_{1} \in \mathbb{R}$ such that for all $x \in K$

$$
\begin{aligned}
& \int_{a}^{b}\left[\eta(t, x, u)^{T} f_{x}(t, u, \dot{u})+\frac{d}{d t} \eta(t, x, u)^{T} f_{\dot{x}}(t, u, \dot{u})\right. \\
& \left.\quad+\rho_{0}\|\theta(t, x, u)\|^{2}\right] d t \geqq 0 \\
& \quad \Longrightarrow b_{0}(x, u)\left\{\int_{a}^{b} f(t, x, \dot{x}) d t-\int_{a}^{b} f(t, u, \dot{u}) d t\right\} \geqq 0,
\end{aligned}
$$

$$
\begin{gathered}
-b_{1}(x, u) \int_{a}^{b} h(t, u, \dot{u}) d t \leqq 0 \\
\Longrightarrow \int_{a}^{b}\left[\eta(t, x, u)^{T} h_{x}(t, u, \dot{u})+\frac{d}{d t} \eta(t, x, u)^{T} h_{\dot{x}}(t, u, \dot{u})\right. \\
\left.\quad+\rho_{1}\|\theta(t, x, u)\|^{2}\right] d t \leqq 0 .
\end{gathered}
$$

If, in the above definition, inequality (16) is satisfied as

$$
\begin{aligned}
& \int_{a}^{b}\left[\eta(t, x, u)^{T} f_{x}(t, u, \dot{u})+\frac{d}{d t} \eta(t, x, u)^{T} f_{\dot{x}}(t, u, \dot{u})\right. \\
& \left.\quad+\rho_{0}\|\theta(t, x, u)\|^{2}\right] d t \geqq 0 \\
& \quad \Longrightarrow b_{0}(x, u)\left\{\int_{a}^{b} f(t, x, \dot{x}) d t-\int_{a}^{b} f(t, u, \dot{u}) d t\right\}>0
\end{aligned}
$$


then we say that a pair $(f, h)$ is $B-\rho_{0}-(\eta, \theta)$-strictly pseudo$\rho_{1}-(\eta, \theta)$-quasi-type-I at $u \in C\left(I, \mathbb{R}^{n}\right)$ with respect to $b_{0}, b_{1}$, $\eta$, and $\theta$ and $\rho_{0}, \rho_{1} \in \mathbb{R}$.

Remark 10. Let $\psi: I \times \mathbb{R}^{n} \times \mathbb{R}^{n} \rightarrow \mathbb{R}$ be a continuously differentiable function with respect to each of the arguments. Let $x, u: I \rightarrow \mathbb{R}^{n}$ be differentiable with $x(a)=u(a)=\alpha$ and $x(b)=u(b)=\beta$. Then,

$\int_{a}^{b} \frac{d}{d t} \eta(t, x, u)^{T} \psi_{\dot{x}}(t, u, \dot{u}) d t=-\int_{a}^{b} \eta(t, x, u) \frac{d}{d t} \psi_{\dot{x}}(t, u, \dot{u}) d t$.

Lemma 11. Every $\left(\rho_{0}, \rho_{1}\right)-(\eta, \theta)$-B-type-I function is $\left(\rho_{0}, \rho_{1}\right)-(\eta, \theta)$-B-strongly pseudo-type-I function but the converse is not true.

Proof. It is clear that the statement every $\left(\rho_{0}, \rho_{1}\right)-(\eta, \theta)-B$ type-I function is $\left(\rho_{0}, \rho_{1}\right)-(\eta, \theta)$ - $B$-strongly pseudo-typeI function but the converse is not true follows from the following counter example.

Example 12. Let $f: I \times[0,1] \times[0,1] \rightarrow \mathbb{R}$ be defined by

$$
f(t, x(t), \dot{x}(t))=-x^{3}(t)-x(t) .
$$

Let $h: I \times[0,1] \times[0,1] \rightarrow \mathbb{R}$ be defined by

$$
h(t, x(t), \dot{x}(t))=3 x^{2}(t)+x(t)-4 .
$$

Let the functions $\eta: I \times[0,1] \times[0,1] \rightarrow \mathbb{R}$ and $\theta: I \times[0,1] \times$ $[0,1] \rightarrow \mathbb{R}$ be defined by

$$
\begin{gathered}
\eta(t, x(t), u(t))= \begin{cases}\frac{x(t)-u(t)}{5} & \text { if } u(t)<x(t), \\
0 & \text { if } u(t) \geq x(\mathrm{t}),\end{cases} \\
\theta(t, x(t), u(t))= \begin{cases}\sqrt{\frac{x(t)-u(t)}{5}} & \text { if } u(t)<x(t), \\
0 & \text { if } u(t) \geq x(t) .\end{cases}
\end{gathered}
$$

Let the functions $b_{0}:[0,1] \times[0,1] \rightarrow \mathbb{R}_{+}$and $b_{1}:[0,1] \times$ $[0,1] \rightarrow \mathbb{R}_{+}$be defined by

$$
b_{0}(x(t), u(t))=x(t)+u(t)+2=b_{1}(x(t), u(t)) .
$$

Taking $\rho_{0}=-1$ and $\rho_{1}=-1$, we have to show that the pair $(f, h)$ is $\left(\rho_{0}, \rho_{1}\right)-(\eta, \theta)$-B-strongly pseudo-type-I but not $\left(\rho_{0}, \rho_{1}\right)-(\eta, \theta)$-B-type-I with respect to $b_{0}, b_{1}, \eta$, and $\theta$.

First we verify that $(f, h)$ is $\left(\rho_{0}, \rho_{1}\right)-(\eta, \theta)$-B-strongly pseudo-type-I function.
Case 1. When $u(t)<x(t)$, it follows that

$$
\begin{aligned}
& b_{0}(x(t), u(t))\left\{\int_{a}^{b} f(t, x, \dot{x}) d t-\int_{a}^{b} f(t, u, \dot{u}) d t\right\} \\
& =(x(t)+u(t)+2)\left[\int_{a}^{b}\left(-x^{3}(t)-x(t)+u^{3}(t)+u(t)\right) d t\right] \\
& =(x(t)+u(t)+2)\left[\int_{a}^{b}\left(\left(u^{3}(t)-x^{3}(t)\right)+(u(t)-x(t))\right) d t\right] \\
& =(x(t)+u(t)+2) \\
& \quad \times\left[\int_{a}^{b}(u(t)-x(t)) \times\left(u^{2}(t)+x^{2}(t)+u(t) x(t)+1\right) d t\right] \\
& <0 \quad(\operatorname{since} x(t), u(t) \in[0,1]) \\
& \Longrightarrow \int_{a}^{b}\left[\eta(t, x(t), u(t))^{T} f_{x}(t, u(t), \dot{u}(t))+\frac{d}{d t} \eta(t, x(t), u(t))^{T}\right. \\
& =\int_{a}^{b}\left(\frac{x(t)-u(t)}{5}\right)\left(-3 u^{2}(t)-2\right) d t \\
& =\int_{a}^{b}\left[\left(\frac{x(t)-u(t)}{5}\right)\left(-3 u^{2}(t)-1\right)-\left(\frac{x(t)-u(t)}{5}\right)\right] d t \\
& \times f_{\dot{x}}\left(t, u(t), \dot{u}(t)+\rho_{0}\|\theta(t, x(t), u(t))\|^{2}\right] d t
\end{aligned}
$$$$
<0 .
$$

Again

$$
\begin{aligned}
\int_{a}^{b} & {\left[\eta(t, x(t), u(t))^{T} h_{x}(t, u(t), \dot{u}(t))+\frac{d}{d t} \eta(t, x(t), u(t))^{T} h_{\dot{x}}\right.} \\
& \left.\times(t, u(t), \dot{u}(t))+\rho_{1}\|\theta(t, x(t), u(t))\|^{2}\right] d t \\
& =\int_{a}^{b}\left[\left(\frac{x(t)-u(t)}{5}\right)(6 u(t)+1)-\left(\frac{x(t)-u(t)}{5}\right)\right] d t \\
& =\int_{a}^{b}\left[\left(\frac{x(t)-u(t)}{5}\right) 6 u(t)\right] d t \\
\geqq & 0 \\
\Longrightarrow & -b_{1}(x(t), u(t)) \int_{a}^{b} h(t, u(t), \dot{u}(t)) d t \\
& =-(x(t)+u(t)+2) \int_{a}^{b}\left(3 u^{2}(t)+u(t)-4\right) d t
\end{aligned}
$$

$\geqq 0$. 
Case 2. When $u(t) \geq x(t)$, it is clear that

$$
\begin{aligned}
& \int_{a}^{b}\left[\eta(t, x(t), u(t))^{T} f_{x}(t, u(t), \dot{u}(t))+\frac{d}{d t} \eta(t, x(t), u(t))^{T}\right. \\
& \left.\quad \times f_{\dot{x}}(t, u(t), \dot{u}(t))+\rho_{0}\|\theta(t, x(t), u(t))\|^{2}\right] d t \geqq 0 \\
& \Longrightarrow b_{0}(x(t), u(t)) \\
& \quad \times\left\{\int_{a}^{b} f(t, x(t), \dot{x}(t)) d t-\int_{a}^{b} f(t, u(t), \dot{u}(t)) d t\right\} \geqq 0, \\
& \int_{a}^{b}\left[\eta(t, x(t), u(t))^{T} h_{x}(t, u(t), \dot{u}(t))+\frac{d}{d t} \eta(t, x(t), u(t))^{T}\right. \\
& \left.\quad \times h_{\dot{x}}(t, u(t), \dot{u}(t))+\rho_{1}\|\theta(t, x(t), u(t))\|^{2}\right] d t \geqq 0 \\
& \Longrightarrow-b_{1}(x(t), u(t)) \int_{a}^{b} h(t, u(t), \dot{u}(t)) d t \geqq 0 .
\end{aligned}
$$

From Cases 1 and 2, it is clear that the pair $(f, h)$ is $\left(\rho_{0}, \rho_{1}\right)-$ $(\eta, \theta)$ - $B$-strongly pseudo-type-I.

But the pair $(f, h)$ is not $\left(\rho_{0}, \rho_{1}\right)-(\eta, \theta)$-B-type-I for $u(t)<$ $x(t)$ because

$$
\begin{gathered}
b_{0}(x(t), u(t))\left\{\int_{a}^{b} f(t, x(t), \dot{x}(t)) d t-\int_{a}^{b} f(t, u(t), \dot{u}(t)) d t\right\} \\
-\left\{\int _ { a } ^ { b } \left[\eta(t, x(t), u(t))^{T} f_{x}(t, u(t), \dot{u}(t))+\frac{d}{d t} \eta(t, x(t), u(t))^{T}\right.\right. \\
\left.\left.\times f_{\dot{x}}(t, u(t), \dot{u}(t))+\rho_{0}\|\theta(t, x(t), u(t))\|^{2}\right] d t\right\} \\
=(x(t)+u(t)+2) \int_{a}^{b}\left\{\left(u^{3}(t)-x^{3}(t)\right)+(u(t)-x(t))\right. \\
=(x(t)+u(t)+2) \\
\times \int_{a}^{b}\left\{\left(\left(\frac{x(t)-u(t)}{5}\right)\right)(u(t)-x(t))\right. \\
\times\left(u^{2}(t)+x^{2}(t)+u(t) x(t)+1\right) \\
\left.+\left(\frac{x(t)-u(t)}{5}\right)\left(3 u^{2}(t)+2\right)\right\} d t
\end{gathered}
$$$$
<0 .
$$

\section{Optimality Conditions}

Theorem 13 (necessary optimality conditions). Let $\stackrel{*}{x} \in K$ be a properly efficient solution for (VP) which is assumed to be normal solution for $\left(P_{k}^{*}\right)$, for $k=1,2, \ldots, p$. Then, there exist

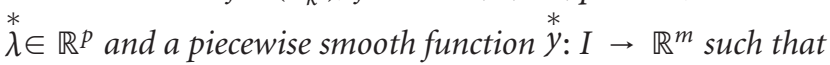

$$
\begin{aligned}
& \stackrel{*}{\lambda}^{T} f_{x}(t, \stackrel{*}{x}, \stackrel{*}{x})+\stackrel{*}{y}(t)^{T} h_{x}(t, \stackrel{*}{x}, \stackrel{*}{x}) \\
& =\frac{d}{d t}\left(\stackrel{*}{\lambda}^{T} f_{\dot{x}}(t, \stackrel{*}{x}, \stackrel{*}{x})+\stackrel{*}{y}^{*}(t)^{T} h_{\dot{x}}(t, \stackrel{*}{x}, \stackrel{*}{x})\right), \\
& \stackrel{*}{y}(t)^{T} h(t, \stackrel{*}{x}, \stackrel{*}{x})=0, \quad t \in I, \\
& \stackrel{*}{y}(t) \geqq 0, \quad t \in I, \\
& \stackrel{*}{\lambda \geqq 0 .}
\end{aligned}
$$

Proof. See [5].

Theorem 14. Let $x^{*}$ be a feasible solution of $(V P)$, and let there exist $\stackrel{*}{\lambda} \in \mathbb{R}^{p}, \stackrel{*}{\lambda}>0$, and a piecewise smooth function $\stackrel{*}{y}: I \rightarrow \mathbb{R}^{m}$ such that for all $t \in I$

$$
\begin{gathered}
\stackrel{*}{\lambda}^{T} f_{x}(t, \stackrel{*}{x}, \stackrel{*}{x})+\stackrel{*}{y}(t)^{T} h_{x}(t, \stackrel{*}{x}, \stackrel{*}{x}) \\
=\frac{d}{d t}\left(\stackrel{*}{\lambda}^{T} f_{\dot{x}}(t, \stackrel{*}{x}, \stackrel{*}{x})+\stackrel{*}{y}(t)^{T} h_{\dot{x}}(t, \stackrel{*}{x}, \stackrel{*}{x})\right), \\
\stackrel{*}{y}(t)^{T} h(t, \stackrel{*}{x}, \stackrel{*}{x})=0, \\
\stackrel{*}{y}(t) \geqq 0 .
\end{gathered}
$$

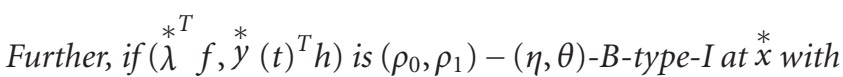
respect to functions $b_{0}, b_{1}, \eta, \theta, \rho_{0}, \rho_{1} \in \mathbb{R}$, and $\rho_{0}+\rho_{1} \geqq 0$, with $b_{0}(x, \stackrel{*}{x})>0$ for all $x \in K$, then $\stackrel{*}{x}$ is a properly efficient solution of $(V P)$.

Proof. As $\left(\stackrel{*}{\lambda}^{T} f, *^{*}(t)^{T} h\right)$ is $\left(\rho_{0}, \rho_{1}\right)-(\eta, \theta)$-B-type-I at $\stackrel{*}{x}$, for all $x \in K$, we have

$$
\begin{aligned}
& b_{0}(x, \stackrel{*}{x})\left\{\int_{a}^{b} *^{T} f(t, x, \dot{x}) d t-\int_{a}^{b} *^{T} f\left(t, x^{*}, \dot{*} x\right) d t\right\} \\
& \geqq \int_{a}^{b}\left[\eta(t, x, \underset{*}{*})^{T} *^{T} f_{x}(t, \underset{*}{*}, \underset{*}{x})\right. \\
& \left.+\frac{d}{d t} \eta(t, x, \stackrel{*}{x})^{T} \stackrel{*}{\lambda}^{T} f_{\dot{x}}(t, \stackrel{*}{x}, \stackrel{*}{x})+\rho_{0}\|\theta(t, x, \stackrel{*}{x})\|^{2}\right] d t, \\
& -b_{1}(x, \stackrel{*}{x}) \int_{a}^{b} \stackrel{*}{y}(t)^{T} h(t, \stackrel{*}{x}, \stackrel{*}{x}) d t \\
& \geqq \int_{a}^{b}\left[\eta(t, x, \stackrel{*}{x})^{T}{ }^{*} y(t)^{T} h_{x}(t, \underset{*}{*}, \underset{x}{x})+\frac{d}{d t} \eta(t, x, \stackrel{*}{x})^{T}\right. \\
& \left.x^{*} y^{*}(t)^{T} h_{\dot{x}}(t, \stackrel{*}{x}, \stackrel{*}{x})+\rho_{1}\|\theta(t, x, \stackrel{*}{x})\|^{2}\right] d t .
\end{aligned}
$$


Since $\stackrel{*}{y}^{(}(t)^{T} h(t, \stackrel{*}{x}, \stackrel{*}{x})=0$, we write $(33)$ as

$$
\begin{aligned}
0 \geqq \int_{a}^{b}[ & \eta(t, x, \stackrel{*}{x})^{T} \stackrel{*}{y}^{\prime}(t)^{T} h_{x}(t, \stackrel{*}{x}, \stackrel{*}{x})+\frac{d}{d t} \eta(t, x, \stackrel{*}{x})^{T} \\
& \left.\times \stackrel{*}{y}(t)^{T} h_{\dot{x}}(t, \stackrel{*}{x}, \underset{*}{x})+\rho_{1}\|\theta(t, x, \stackrel{*}{x})\|^{2}\right] d t .
\end{aligned}
$$

Adding (32) and (34), we have

$$
\begin{gathered}
b_{0}(x, \stackrel{*}{x})\left\{\int_{a}^{b} *^{T} f(t, x, \dot{x}) d t-\int_{a}^{b} *^{T} f(t, \stackrel{*}{x}, \stackrel{*}{x}) d t\right\} \\
\geqq \int_{a}^{b}\left[\eta(t, x, \stackrel{*}{x})^{T}\left(\stackrel{*^{T}}{\lambda} f_{x}(t, \stackrel{*}{x}, \stackrel{*}{x})+\stackrel{*}{y}(t)^{T} h_{x}(t, \stackrel{*}{x}, \underset{*}{x})\right)\right. \\
+\frac{d}{d t} \eta(t, x, \stackrel{*}{x})^{T} \\
\quad \times\left(\stackrel{*^{T}}{\lambda} f_{\dot{x}}(t, \stackrel{*}{x}, \stackrel{*}{x})+\stackrel{*}{y}(t)^{T} h_{\dot{x}}(t, \stackrel{*}{x}, \underset{*}{x})\right) \\
\left.+\left(\rho_{0}+\rho_{1}\right)\|\theta(t, x, \stackrel{*}{x})\|^{2}\right] d t .
\end{gathered}
$$

Using Remark 10, (35) becomes

$$
\begin{aligned}
& b_{0}(x, \stackrel{*}{x})\left\{\int_{a}^{b} \stackrel{*}{\lambda}^{T} f(t, x, \dot{x}) d t-\int_{a}^{b} *^{T} f(t, \dot{*}, \dot{*} x) d t\right\} \\
& \geqq \int_{a}^{b}\left[\eta ( t , x , \stackrel { * } { x } ) ^ { T } \left\{\left(\stackrel{*}{T}^{T} f_{x}(t, \stackrel{*}{x}, \stackrel{*}{x})+\stackrel{*}{y}(t)^{T} h_{x}(t, \stackrel{*}{x}, \stackrel{*}{x})\right)\right.\right. \\
& \left.-\frac{d}{d t}\left(\stackrel{*}{\lambda}^{T} f_{\dot{x}}(t, \stackrel{*}{x}, \stackrel{*}{x})+\stackrel{*}{y}(t)^{T} h_{\dot{x}}(t, \stackrel{*}{x}, \stackrel{*}{x})\right)\right\} \\
& \left.+\left(\rho_{0}+\rho_{1}\right)\|\theta(t, x, \stackrel{*}{x})\|^{2}\right] d t
\end{aligned}
$$

By (29) and $\rho_{0}+\rho_{1} \geqq 0$, we have

$$
b_{0}(x, \stackrel{*}{x})\left\{\int_{a}^{b} *^{T} f(t, x, \dot{x}) d t-\int_{a}^{b} *^{T} f(t, \stackrel{*}{x}, \stackrel{*}{x}) d t\right\} \geqq 0
$$

As $b_{0}(x, \stackrel{*}{x})>0$, it follows that

$$
\int_{a}^{b} \stackrel{*^{T}}{\lambda} f(t, x, \dot{x}) d t-\int_{a}^{b} \stackrel{*}{T}^{T} f(t, \stackrel{*}{x}, \stackrel{*}{x}) d t \geqq 0,
$$

that is,

$$
\int_{a}^{b} \stackrel{*^{T}}{\lambda} f(t, x, \dot{x}) d t \geqq \int_{a}^{b} \stackrel{*}{T}^{T} f(t, \stackrel{*}{x}, \stackrel{*}{x}) d t
$$

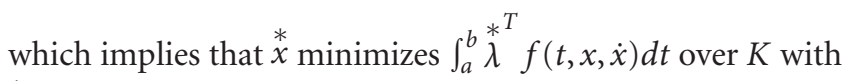
$\stackrel{*}{\lambda}>0$. Hence $\stackrel{*}{x}$ is a properly efficient solution of $(V P)$ on account of Theorem 1 of Bector and Husain [3].
Theorem 15. Let $\stackrel{*}{x}$ be a feasible solution of $(V P)$, and let there exist $\stackrel{*}{\lambda} \in \mathbb{R}^{p}, \stackrel{*}{\lambda}>0$, and a piecewise smooth function $\stackrel{*}{y}: I \rightarrow \mathbb{R}^{m}$ such that, for every $t \in I$, conditions, (29)-(31) of Theorem 14 are satisfied. Further, if $\left(\stackrel{*}{T}^{T} f,{ }^{*}(t)^{T} h\right)$ is $\left(\rho_{0}, \rho_{1}\right)-(\eta, \theta)-B-$ semistrictly-type-I at $\stackrel{*}{x}$ with respect to functions $b_{0}, b_{1}, \eta, \theta$, $\rho_{0}, \rho_{1} \in \mathbb{R}$, and $\rho_{0}+\rho_{1} \geqq 0$, with $b_{0}\left(x,{ }^{*} x\right)>0$ for all $x \in K$, then $\stackrel{*}{x}$ is a properly efficient solution of $(V P)$.

Proof. Let $\stackrel{*}{x}$ be not; an efficient solution of $(V P)$, then there exist $x \in K$ and an index $1 \leqq r \leqq P$ such that the following inequalities

$$
\begin{gathered}
\int_{a}^{b} f_{j}(t, x, \dot{x}) d t \leqq \int_{a}^{b} f_{j}(t, \stackrel{*}{x}, \stackrel{*}{x}) d t, \quad \forall j \neq r, j=1,2, \ldots p \\
\int_{a}^{b} f_{r}(t, x, \dot{x}) d t<\int_{a}^{b} f_{r}(t, \stackrel{*}{x}, \stackrel{*}{x}) d t
\end{gathered}
$$

hold. Because $\stackrel{*}{\lambda}>0$ and $b_{0}(x, \stackrel{*}{x})>0,(40)$ imply

$$
b_{0}(x, \stackrel{*}{x})\left\{\int_{a}^{b} \stackrel{*}{T}^{T} f(t, x, \dot{x}) d t-\int_{a}^{b} \stackrel{*}{T}^{T} f(t, \stackrel{*}{x}, \stackrel{*}{x}) d t\right\} \leqq 0
$$

Further in view of (30)

$$
b_{1}(x, \stackrel{*}{x}) \int_{a}^{b} \stackrel{*}{y}(t)^{T} h(t, \stackrel{*}{x}, \stackrel{*}{x}) d t=0
$$

As $\left(\stackrel{*}{\lambda}^{T} f, \stackrel{*}{y}^{\prime}(t)^{T} h\right)$ is $\left(\rho_{0}, \rho_{1}\right)-(\eta, \theta)$-B-semistrictly-type-I at ${ }^{*}$ with respect to functions $b_{0}, b_{1}, \eta$, and $\theta$, we have

$$
\begin{aligned}
& \int_{a}^{b}\left[\eta(t, x, \stackrel{*}{x})^{T} \stackrel{*^{T}}{\lambda} f_{x}(t, \stackrel{*}{x}, \stackrel{*}{x})+\frac{d}{d t} \eta(t, x, \stackrel{*}{x})^{T} \stackrel{*^{T}}{\lambda} f_{\dot{x}}(t, \stackrel{*}{x}, \stackrel{*}{x})\right. \\
& \left.+\rho_{0}\|\theta(t, x, \stackrel{*}{x})\|^{2}\right] d t<0 \\
& \int_{a}^{b}\left[\eta(t, x, \stackrel{*}{x})^{T} \stackrel{*}{y}(t)^{T} h_{x}(t, \stackrel{*}{x}, \stackrel{*}{x})+\frac{d}{d t} \eta(t, x, \stackrel{*}{x})^{T}\right. \\
& \left.\times \stackrel{*}{y}(t)^{T} h_{\dot{x}}(t, \stackrel{*}{x}, \stackrel{*}{x})+\rho_{1}\|\theta(t, x, \stackrel{*}{x})\|^{2}\right] d t \leqq 0
\end{aligned}
$$

Adding (43), we have

$$
\begin{aligned}
& \int_{a}^{b}\left\{\eta(t, x, \stackrel{*}{x})^{T}\left[\stackrel{*^{T}}{\lambda} f_{x}(t, \stackrel{*}{x}, \stackrel{*}{x})+\stackrel{*}{y}(t)^{T} h_{x}(t, \stackrel{*}{x}, \stackrel{*}{x})\right]\right. \\
& +\frac{d}{d t} \eta(t, x, \stackrel{*}{x})^{T}\left[\stackrel{*}{\lambda}^{T} f_{\dot{x}}(t, \stackrel{*}{x}, \stackrel{*}{x})+\stackrel{*}{y}^{*}(t)^{T} h_{\dot{x}}(t, \stackrel{*}{x}, \stackrel{*}{x})\right] \\
& \left.+\left(\rho_{0}+\rho_{1}\right)\|\theta(t, x, \underset{*}{x})\|^{2}\right\} d t<0 .
\end{aligned}
$$


By Remark 10, we have

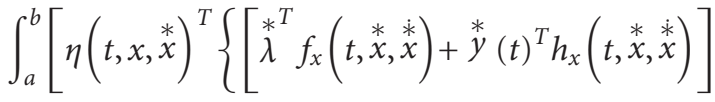

$$
\begin{aligned}
& \left.-\frac{d}{d t}\left[\stackrel{*}{\lambda}^{T} f_{\dot{x}}(t, \stackrel{*}{x}, \stackrel{*}{x})+\stackrel{*}{y}(t)^{T} h_{\dot{x}}(t, \stackrel{*}{x}, \stackrel{*}{x})\right]\right\} \\
& \left.+\left(\rho_{0}+\rho_{1}\right)\|\theta(t, x, \stackrel{*}{x})\|^{2}\right] d t<0,
\end{aligned}
$$

which contradicts $(29)$ and $\rho_{0}+\rho_{1} \geq 0$. Hence $\stackrel{*}{x}$ is a properly efficient solution of $(V P)$.

Theorem 16. Let $\stackrel{*}{x}$ be a feasible solution of (VP), and let there exist $\stackrel{*}{\lambda} \in \mathbb{R}^{p}, \stackrel{*}{\lambda}>0$, and a piecewise smooth function

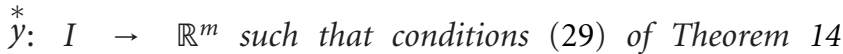
are satisfied by $(\stackrel{*}{x}, \stackrel{*}{\lambda}, \stackrel{*}{y})$. Further, if $\left(\stackrel{*}{\lambda}^{T} f, *^{*}(t)^{T} h\right)$ is $B-$ $\rho_{0}-(\eta, \theta)$-strongly pseudo- $\rho_{1}-(\eta, \theta)$-quasi-type-I at ${ }_{x}^{*}$ with respect to functions $b_{0}, b_{1}, \eta, \theta, \rho_{0}, \rho_{1} \in \mathbb{R}$ and $\rho_{0}+\rho_{1} \geqq$ $0, b_{1}(x, *)>0$ for all $x \in K$, then ${ }^{*}$ is a properly efficient solution of $(V P)$.

Proof. From (30), we have

$$
b_{1}(x, \stackrel{*}{x}) \int_{a}^{b} \stackrel{*}{y}(t)^{T} h(t, \stackrel{*}{x}, \stackrel{*}{x})=0 .
$$

As $\left(\stackrel{*}{\lambda}^{T} f, \stackrel{*}{y}^{\prime}(t)^{T} h\right)$ is $B-\rho_{0}-(\eta, \theta)$-strongly pseudo- $\rho_{1}-(\eta, \theta)$ quasi-type-I at ${ }_{x}^{*}$ with respect to functions $b_{0}, b_{1}, \eta$, and $\theta$,

$$
\begin{aligned}
& \int_{a}^{b}\left[\eta(t, x, \stackrel{*}{x})^{T} \stackrel{*}{y}(t)^{T} h_{x}(t, \stackrel{*}{x}, \dot{*} x)+\frac{d}{d t} \eta(t, x, \stackrel{*}{x})^{T} \stackrel{*}{y}(t)^{T}\right. \\
& \left.\times h_{\dot{x}}(t, \stackrel{*}{x}, \stackrel{*}{x})+\rho_{1}\|\theta(t, x, \stackrel{*}{x})\|^{2}\right] d t \leqq 0 .
\end{aligned}
$$

Using Remark 10, (45) becomes

$$
\begin{gathered}
\int_{a}^{b}\left\{\eta(t, x, \underset{*}{x})^{T}\left[\stackrel{*}{y}(t)^{T} h_{x}(t, \stackrel{*}{x}, \underset{*}{x})-\frac{d}{d t} \stackrel{*}{y}^{*}(t)^{T} h_{\dot{x}}(t, \underset{*}{*}, \underset{*}{x})\right]\right. \\
\left.+\rho_{1}\|\theta(t, \underset{*}{*})\|^{2}\right\} d t \leqq 0 .
\end{gathered}
$$

Equation (48) along with (29) gives

$$
\begin{aligned}
& \int_{a}^{b}\left\{\eta(t, x, \stackrel{*}{x})^{T}\left[\stackrel{*}{T}^{T} f_{x}(t, \stackrel{*}{x}, \stackrel{*}{x})-\frac{d}{d t} \lambda^{*^{T}} f_{\dot{x}}(t, \stackrel{*}{x}, \stackrel{*}{x})\right]\right. \\
& \left.-\rho_{1}\|\theta(t, x, \stackrel{*}{x})\|^{2}\right\} d t \geqq 0 .
\end{aligned}
$$

Using Remark 10, for all $x \in K$, (48) becomes

$$
\begin{gathered}
\int_{a}^{b}\left[\eta(t, x, \stackrel{*}{x})^{T} \stackrel{*^{T}}{\lambda} f_{x}(t, \stackrel{*}{x}, \underset{*}{x})+\frac{d}{d t} \eta(t, x, \stackrel{*}{x})^{T} \stackrel{*^{T}}{\lambda} f_{\dot{x}}(t, \stackrel{*}{x}, \underset{*}{x})\right. \\
\left.-\rho_{1}\|\theta(t, \stackrel{*}{x})\|^{2}\right] d t \geqq 0 \quad \forall x \in K .
\end{gathered}
$$

As $\rho_{0}+\rho_{1} \geqq 0$, we have

$$
\begin{gathered}
\int_{a}^{b}\left[\eta(t, x, \stackrel{*}{x})^{T} \stackrel{*^{T}}{\lambda} f_{x}(t, \stackrel{*}{x}, \underset{*}{x})+\frac{d}{d t} \eta(t, x, \stackrel{*}{x})^{T} \stackrel{*^{T}}{\lambda} f_{\dot{x}}(t, \stackrel{*}{x}, \stackrel{*}{x})\right. \\
\left.+\rho_{0}\|\theta(t, x, \stackrel{*}{x})\|^{2}\right] d t \geqq 0 .
\end{gathered}
$$

Equation (51) along with the fact that $\left(\stackrel{*}{\lambda}^{T} f, \stackrel{*}{y}^{\prime}(t)^{T} h\right)$ is $B-$ $\rho_{0}-(\eta, \theta)$-strongly pseudo- $\rho_{1}-(\eta, \theta)$-quasi-type-I at $\stackrel{*}{x}$ gives

$$
b_{0}(x, \stackrel{*}{x})\left\{\int_{a}^{b} *^{T} f(t, x, \dot{x})-\int_{a}^{b} *^{T} f(t, \stackrel{*}{x}, \stackrel{*}{x})\right\} \geqq 0 .
$$

Since $b_{0}(x, \stackrel{*}{x})>0$, it follows that

$$
\int_{a}^{b} \stackrel{*}{\lambda}^{T} f(t, x, \dot{x}) \geqq \int_{a}^{b} \stackrel{*}{T}^{T} f(t, \stackrel{*}{x}, \stackrel{*}{x}) \quad \forall x \in K .
$$

Hence $\stackrel{*}{x}$ minimizes $\int_{a}^{b} \stackrel{*}{\lambda}^{T} f(t, x, \dot{x}) d t$ over $K$ with $\stackrel{*}{\lambda}>0$.

Therefore $\stackrel{*}{x}$ is a properly efficient solution of (VP) ([3, Theorem 1]).

Theorem 17. Let $\stackrel{*}{x}$ be a feasible solution of $(V P)$, and let there exist $\stackrel{*}{\lambda} \in \mathbb{R}^{p}, \stackrel{*}{\lambda}>0$, and a piecewise smooth function $\stackrel{*}{y}: I \rightarrow \mathbb{R}^{m}$ such that the conditions (29)-(31) of Theorem 14 are satisfied by $(* \underset{x}{*} \lambda, \stackrel{*}{y})$. Further, if $\left(*^{T} f, *^{*}(t)^{T} h\right)$ is $B-\rho_{0}-(\eta, \theta)$-quasi$\rho_{1}-(\eta, \theta)$-strictly pseudo-type-I at ${ }_{x}^{*}$ with respect to functions $b_{0}, b_{1}, \eta, \theta, \rho_{0}, \rho_{1} \in \mathbb{R}$, and $\rho_{0}+\rho_{1} \geqq 0$, with $b_{0}(x, \stackrel{*}{x})>0$ for all $x \in K$, then $\stackrel{*}{x}$ is a properly efficient solution of $(V P)$.

Proof. If ${ }^{*}$ is not an efficient solution of $(V P)$, then there exist $x \in K$ and an index $r, 1 \leq r \leqq p$, such that

$$
\begin{gathered}
\int_{a}^{b} f_{j}(t, x, \dot{x}) d t \leqq \int_{a}^{b} f_{j}(t, \stackrel{*}{x}, \underset{*}{x}) d t \quad \forall j \neq r, j=1,2, \ldots p, \\
\int_{a}^{b} f_{r}(t, x, \dot{x}) d t<\int_{a}^{b} f_{r}(t, \stackrel{*}{x}, \underset{*}{x}) d t .
\end{gathered}
$$

Because $\stackrel{*}{\lambda}>0$ and $b_{0}(x, \stackrel{*}{x})>0$, the previous relations give

$$
b_{0}(x, \stackrel{*}{x})\left\{\int_{a}^{b} *^{T} f(t, x, \dot{x}) d t-\int_{a}^{b} *^{T} f(t, \stackrel{*}{x}, \dot{*} x) d t\right\} \leqq 0,
$$


which along with the fact that $\left(\stackrel{*}{\lambda}^{T} f, *^{*}(t)^{T} h\right)$ is $B-\rho_{0}-(\eta, \theta)$ quasi- $\rho_{1}-(\eta, \theta)$-strictly pseudo-type-I at ${ }_{x}^{*}$ with respect to functions $b_{0}, b_{1}, \eta$, and $\theta$ gives

$$
\begin{aligned}
& \int_{a}^{b}\left[\eta(t, x, \stackrel{*}{x})^{T} \stackrel{*}{\lambda}^{T} f_{x}(t, \stackrel{*}{x}, \stackrel{*}{x})+\frac{d}{d t} \eta(t, x, \stackrel{*}{x})^{T} \stackrel{*}{\lambda}^{T} f_{\dot{x}}(t, \stackrel{*}{x}, \stackrel{*}{x})\right. \\
& \left.+\rho_{0}\|\theta(t, x, \stackrel{*}{x})\|^{2}\right] d t \leqq 0 .
\end{aligned}
$$

Using Remark 10, (56) becomes

$$
\begin{aligned}
& \int_{a}^{b}\left[\eta(t, x, \stackrel{*}{x})^{T} \stackrel{*}{\lambda}^{T} f_{x}(t, \stackrel{*}{*}, \underset{*}{x})-\eta(t, x, \stackrel{*}{x})^{T} \frac{d}{d t} \lambda^{*^{T}} f_{\dot{x}}(t, \stackrel{*}{x}, \underset{*}{x})\right. \\
& \left.+\rho_{0}\|\theta(t, x, \stackrel{*}{x})\|^{2}\right] d t \leqq 0 .
\end{aligned}
$$

that is,

$$
\begin{aligned}
& \int_{a}^{b}\left\{\eta(t, x, \stackrel{*}{x})^{T}\left[\stackrel{*}{\lambda}^{T} f_{x}(t, \stackrel{*}{x}, \stackrel{*}{x})-\frac{d}{d t} \lambda^{*^{T}} f_{\dot{x}}(t, \stackrel{*}{x}, \stackrel{*}{x})\right]\right. \\
& \left.+\rho_{0}\|\theta(t, x, \stackrel{*}{x})\|^{2}\right\} d t \leqq 0
\end{aligned}
$$

Now using (29) in (58), we have

$$
\begin{aligned}
\int_{a}^{b}\{ & -(\eta(t, x, \stackrel{*}{x}))^{T} \\
& \times\left[{ }^{*} y(t)^{T} h_{x}(t, \stackrel{*}{x}, \stackrel{*}{x})-\frac{d}{d t} \stackrel{*}{y}_{(t)^{T}} h_{\dot{x}}(t, \stackrel{*}{x}, \underset{*}{x})\right] d t \\
& \left.+\rho_{0}\|\theta(t, x, \stackrel{*}{x})\|^{2}\right\} \leqq 0 .
\end{aligned}
$$

It follows that

$$
\begin{aligned}
& \int_{a}^{b}\left\{\eta(t, x, \stackrel{*}{x})^{T}\left[\stackrel{*}{y}(t)^{T} h_{x}(t, \stackrel{*}{x}, \underset{*}{x})-\frac{d}{d t} \stackrel{*}{y}^{*}(t)^{T} h_{\dot{x}}(t, \stackrel{*}{x}, \stackrel{*}{\mathrm{x}})\right]\right. \\
& \left.-\rho_{0}\|\theta(t, x, \stackrel{*}{x})\|^{2}\right\} d t \geqq 0, \\
& \Longrightarrow \int_{a}^{b} \eta(t, x, \stackrel{*}{x})^{T}\left[\stackrel{*}{y}(t)^{T} h_{x}(t, \stackrel{*}{x}, \stackrel{*}{x})-\frac{d}{d t} \stackrel{*}{y}(t)^{T} h_{\dot{x}}(t, \stackrel{*}{x}, \stackrel{*}{x})\right] d t \\
& \geqq \rho_{0}\|\theta(t, x, \stackrel{*}{x})\|^{2} \text {. }
\end{aligned}
$$

As $\rho_{0}+\rho_{1} \geqq 0$, we have

$$
\begin{aligned}
& \int_{a}^{b}\left\{\eta(t, x, \stackrel{*}{x})^{T}\left[\stackrel{*}{y}(t)^{T} h_{x}(t, \stackrel{*}{x}, \underset{*}{x})-\frac{d}{d t} \stackrel{*}{y}^{*}(t)^{T} h_{\dot{x}}(t, \stackrel{*}{x}, \underset{*}{x})\right]\right. \\
& \left.+\rho_{1}\|\theta(t, x, \stackrel{*}{x})\|^{2}\right\} d t \geqq 0 .
\end{aligned}
$$

Again using Remark 10, we obtain

$$
\begin{aligned}
& \int_{a}^{b}\left[\eta(t, x, \stackrel{*}{x})^{T} \stackrel{*}{y}(t)^{T} h_{x}(t, \stackrel{*}{x}, \underset{*}{x})+\frac{d}{d t} \eta(t, x, \stackrel{*}{x})^{T}{ }^{*} y(t)^{T}\right. \\
& \left.\times h_{\dot{x}}(t, \stackrel{*}{x}, \stackrel{*}{x})+\rho_{1}\|\theta(t, x, \stackrel{*}{x})\|^{2}\right] d t \geqq 0,
\end{aligned}
$$

which in view of a given hypothesis implies that

$$
-b_{1}(x, \stackrel{*}{x}) \int_{a}^{b} \stackrel{*}{y}(t)^{T} h(t, \stackrel{*}{x}, \underset{*}{x})>0 .
$$

Since $b_{1}(x, \stackrel{*}{x})>0$, we obtain

$$
-\int_{a}^{b} \stackrel{*}{y}(t)^{T} h(t, \stackrel{*}{x}, \underset{*}{x})>0
$$

which contradicts (30). Hence $\stackrel{*}{x}$ is a properly efficient solution of $(V P)$.

\section{Duality}

The Mond-Weir-type dual problem associated with $(V P)$ is given by

$$
\begin{array}{ll}
(V D) \text { maximize } & \int_{a}^{b} f(t, u, \dot{u}) d t \\
& =\left(\int_{a}^{b} f_{1}(t, u, \dot{u}) d t, \ldots, \int_{a}^{b} f_{p}(t, u, \dot{u}) d t\right),
\end{array}
$$

subject to $u(a)=\alpha, u(b)=\beta$,

$$
\begin{aligned}
& \lambda^{T} f_{x}(t, u, \dot{u})+y(t)^{T} h_{x}(t, u, \dot{u}) \\
& =\frac{d}{d t}\left(\lambda^{T} f_{\dot{x}}(t, u, \dot{u})+y(t)^{T} h_{\dot{x}}(t, u, \dot{u})\right), \\
& y(t)^{T} h(t, u, \dot{u}) \geqq 0, \quad t \in I, \\
& y(t) \geqq 0, \quad t \in I, \\
& \lambda \in \mathbb{R}^{p}, \quad \lambda \geqq 0, \quad \lambda^{T} e=1, \\
& \quad e=(1,1, \ldots, 1) \in \mathbb{R}^{p} .
\end{aligned}
$$

We now establish duality results between $(V P)$ and $(V D)$ under generalized $B$-type-I conditions.

Theorem 18 (weak duality). Let $x$ be a feasible solution of $(V P)$, and let $(u, \lambda, y)$ be a feasible solution of $(V D)$. Let either the following conditions hold.

(i) $\left(\lambda^{T} f, y(t)^{T} h\right)$ is $\left(\rho_{0}, \rho_{1}\right)-(\eta, \theta)-B$-semi-strictly-type$I$ at $u$ with respect to functions $b_{0}, b_{1}, \eta, \theta, \rho_{0}, \rho_{1} \in \mathbb{R}$ and $\rho_{0}+\rho_{1} \geqq 0$.

(ii) $\lambda>0$ and $\left(\lambda^{T} f, y(t)^{T} h\right)$ is $B-\rho_{0}-(\eta, \theta)$-strongly pseudo- $\rho_{1}-(\eta, \theta)$-quasi-type-I at $u$ with respect to functions $b_{0}, b_{1}, \eta$, and $\theta$ with $b_{1}(x, u)>0, \rho_{0}, \rho_{1} \in \mathbb{R}$ and $\rho_{0}+\rho_{1} \geqq 0$. 
(iii) $\left(\lambda^{T} f, y(t)^{T} h\right)$ is $B-\rho_{0}-(\eta, \theta)-q u a s i-\rho_{1}-(\eta, \theta)$-strictly pseudo-type-I at $u$ with respect to functions $b_{0}, b_{1}, \eta$, and $\theta$, with $b_{0}(x, u)>0, \rho_{0}, \rho_{1} \in \mathbb{R}$, and $\rho_{0}+\rho_{1} \geqq 0$, Then

$$
\int_{a}^{b} f(t, x, \dot{x}) d t \int_{a}^{b} f(t, u, \dot{u}) d t .
$$

Proof. (i) Let, if possible,

$$
\int_{a}^{b} f(t, x, \dot{x}) d t \leq \int_{a}^{b} f(t, u, \dot{u}) d t .
$$

Then there exists an index $r, 1 \leqq r \leqq p$, such that

$$
\begin{gathered}
\int_{a}^{b} f_{j}(t, x, \dot{x}) d t \leqq \int_{a}^{b} f_{j}(t, \stackrel{*}{*}, \dot{*}) d t \quad \forall j \neq r, j=1,2, \ldots p, \\
\int_{a}^{b} f_{r}(t, x, \dot{x}) d t<\int_{a}^{b} f_{r}(t, \stackrel{*}{x}, \underset{x}{x}) d t .
\end{gathered}
$$

Because $\lambda>0$ and $b_{0}(x, u)>0$, the above relations give

$$
b_{0}(x, u) \int_{a}^{b} \lambda^{T} f(t, x, \dot{x}) d t \leqq b_{0}(x, u) \int_{a}^{b} \lambda^{T} f(t, u, \dot{u}) d t,
$$

that is,

$$
b_{0}(x, u)\left[\int_{a}^{b} \lambda^{T} f(t, x, \dot{x}) d t-\int_{a}^{b} \lambda^{T} f(t, u, \dot{u}) d t\right] \leqq 0
$$

which is the same as (41) with $\stackrel{*}{x}$ replaced by $u$ and $\stackrel{*}{\lambda}$ replaced by $\lambda$. The rest of the proof runs on the same line of that of Theorem 15 and hence is omitted.

(ii) From (67), we get

$$
-\int_{a}^{b} y(t)^{T} h(t, u, \dot{u}) \leqq 0 .
$$

Since $b_{1}(x, u)>0$, we have

$$
-b_{1}(x, u) \int_{a}^{b} y(t)^{T} h(t, u, \dot{u}) \leqq 0,
$$

which implies that

$$
\begin{gathered}
\int_{a}^{b}\left[\eta(t, x, u)^{T} y(t)^{T} h_{x}(t, u, \dot{u})+\frac{d}{d t} \eta(t, x, u)^{T} y(t)^{T}\right. \\
\left.\times h_{\dot{x}}(t, u, \dot{u})+\rho_{1}\|\theta(t, x, u)\|^{2}\right] d t \leqq 0
\end{gathered}
$$

on account of hypothesis (ii). This is the same as (45) with $\stackrel{*}{*}$ replaced by $u$ and $\stackrel{*}{y}$ replaced by $y$. Again proceeding on the lines of Theorem 16, we get the result.

(iii) Proof of this part follows on the lines of Theorem 17 and hence is omitted.
Theorem 19 (strong duality). Let $\stackrel{*}{x}$ be a properly efficient solution of $(V P)$. Assume that ${ }^{*}$ is normal for each $\left(P_{k}^{*}\right)$, $k=1,2, \ldots, p$. Then there exist $\stackrel{*}{\lambda} \in \mathbb{R}^{p}$ and a piecewise smooth function $\stackrel{*}{y}: I \rightarrow \mathbb{R}^{m}$ such that $(\stackrel{*}{x}, \stackrel{*}{\lambda}, \stackrel{*}{y})$ is feasible for $(V D)$. Further, if, for each feasible $(u, \lambda, y)$ of $(V D)$, any of the conditions of Theorem 18 hold, then $(\underset{x}{*}, \stackrel{*}{*}, y)$ is a properly efficient solution of (VD).

Proof. Because $\underset{x}{*}$ is a properly efficient solution of $(V P)$, it follows that, from Theorem 13 there exist $\lambda_{\in}^{*} \in \mathbb{R}^{p}$ and a piecewise smooth function ${ }^{*}: I \rightarrow \mathbb{R}^{m}$ such that (28) hold. Moreover, $\underset{x}{*} \in K$, and hence the feasibility of $(\stackrel{*}{x}, \stackrel{*}{\lambda}, \stackrel{*}{y})$ for $(V D)$ follows. As the weak duality (Theorem 18) holds between $(V P)$ and $(V D),(\stackrel{*}{x}, \stackrel{*}{\lambda}, \stackrel{*}{y})$ is an efficient solution of $(V D)$. If $\left(\stackrel{*}{x}, \stackrel{*}{\lambda}, y^{*}\right)$ is not a properly efficient solution of $(V D)$, then, proceeding on the lines to that of [3, Theorem 1], we get a contradiction to the weak duality.

Theorem 20 (strict converse duality). Let $\stackrel{*}{x}$ be feasible for $(V P)$, and let $(\stackrel{*}{*}, \stackrel{*}{\lambda}, y)$ be feasible for $(V D)$ such that

$$
\int_{a}^{b} \stackrel{*}{T}^{T} f(t, \underset{*}{*}, \underset{*}{x}) d t=\int_{a}^{b} \stackrel{*}{T}^{T} f(t, \stackrel{*}{u}, \stackrel{*}{u}) d t
$$

Further, let $\left(*^{T} f, \stackrel{*}{y}^{\prime}(t)^{T} h\right)$ be $B-\rho_{0}-(\eta, \theta)$-strictly pseudo$\rho_{1}-(\eta, \theta)$-quasi-type-I at $\stackrel{*}{u}$ with respect to functions $b_{0}, b_{1}, \eta$, $\theta, \rho_{0}, \rho_{1} \in \mathbb{R}$, and $\rho_{0}+\rho_{1} \geqq 0, b_{0}(\stackrel{*}{x}, \stackrel{*}{u})>0, b_{1}(\stackrel{*}{x}, \stackrel{*}{u})>0$ for all $\stackrel{*}{x} \in K$. Then $\stackrel{*}{x}$ is a properly efficient solution of $(V P)$.

Proof. We assume that $\stackrel{*}{x} \neq \stackrel{*}{*}$ and get a contradiction. Feasibility of $\left(\stackrel{*}{\mathcal{H}}, \stackrel{*}{\lambda}, \stackrel{*}{y}^{\prime}\right)$ for $(V D)$ implies that

$$
-\int_{a}^{b}{ }^{*}(t)^{T} h(t, \stackrel{*}{u}, \stackrel{*}{u}) d t \leqq 0 .
$$

As $b_{1}(\stackrel{*}{x}, \stackrel{*}{u})>0$, we have

$$
-b_{1}(\stackrel{*}{x}, \stackrel{*}{u}) \int_{a}^{b} \stackrel{*}{y}(t)^{T} h(t, \stackrel{*}{u}, \stackrel{*}{u}) d t \leqq 0 .
$$

Equation (80) along with the fact that $\left(\stackrel{*}{\lambda}^{T} f, \stackrel{*}{y}^{\prime}(t)^{T} h\right)$ is $B-$ $\rho_{0}-(\eta, \theta)$-strictly pseudo- $\rho_{1}-(\eta, \theta)$-quasi-type-I at $\stackrel{*}{u}$ implies that

$$
\begin{aligned}
& \int_{a}^{b}\left[\eta(t, \stackrel{*}{x}, \stackrel{*}{u})^{T} \stackrel{*}{y}(t)^{T} h_{x}(t, \stackrel{*}{u}, \stackrel{*}{u})\right. \\
& \left.+\frac{d}{d t} \eta(t, \stackrel{*}{*}, \stackrel{*}{u})^{T} \stackrel{*}{y}(t)^{T} h_{\dot{x}}(t, \stackrel{*}{u}, \stackrel{*}{u})+\rho_{1}\|\theta(t, \stackrel{*}{x}, \stackrel{*}{u})\|^{2}\right] d t \\
& \leqq 0 \text {. }
\end{aligned}
$$


Using Remark 10 (with $\psi$ replaced by $\left.{ }^{*}(t)^{T} h\right)$ in $(81$ ), we get

$$
\begin{aligned}
& \int_{a}^{b}\left\{\eta(t, \stackrel{*}{x}, \stackrel{*}{u})^{T}\left[\stackrel{*}{y}(t)^{T} h_{x}(t, \stackrel{*}{u}, \stackrel{*}{u})-\frac{d}{d t} \stackrel{*}{y}(t)^{T} h_{\dot{x}}(t, \stackrel{*}{u}, \stackrel{*}{u})\right]\right. \\
& \left.\quad+\rho_{1}\|\theta(t, \stackrel{*}{x}, \stackrel{*}{u})\|^{2}\right\} \leqq 0 .
\end{aligned}
$$

Equation (82) along with (66) gives

$$
\begin{gathered}
\int_{a}^{b}\left\{\eta(t, \stackrel{*}{x}, \stackrel{*}{u})^{T}\left[\stackrel{*}{\lambda}^{T} f_{x}(t, \stackrel{*}{u}, \stackrel{*}{u})-\frac{d}{d t} \stackrel{*}{T}^{T} f_{\dot{x}}(t, \stackrel{*}{u}, \stackrel{*}{u})\right]\right. \\
\left.-\rho_{1}\|\theta(t, \stackrel{*}{x}, \stackrel{*}{u})\|^{2}\right\} \geqq 0 .
\end{gathered}
$$

Since $\rho_{0}+\rho_{1} \geqq 0$, we have

$$
\begin{aligned}
& \int_{a}^{b}\left\{\eta(t, \stackrel{*}{x}, \stackrel{*}{u})^{T}\left[\stackrel{*}{\lambda}^{T} f_{x}(t, \stackrel{*}{u}, \stackrel{*}{u})-\frac{d}{d t} \lambda^{*^{T}} f_{\dot{x}}(t, \stackrel{*}{u}, \stackrel{*}{u})\right]\right. \\
& \left.+\rho_{0}\|\theta(t, \stackrel{*}{x}, \stackrel{*}{u})\|^{2}\right\} d t \geqq 0 .
\end{aligned}
$$

Again using Remark 10 (with $\psi$ replaced by $\lambda^{*^{T}} f$ ) in (84), we obtain

$$
\begin{aligned}
& \int_{a}^{b}\left[\eta(t, \stackrel{*}{*}, \stackrel{*}{u})^{T} \stackrel{*^{T}}{\lambda} f_{x}(t, \stackrel{*}{u}, \stackrel{*}{u})+\frac{d}{d t} \eta\left(t, \stackrel{*}{x}, *^{u}\right)^{T} *^{* T} f_{\dot{x}}(t, \stackrel{*}{u}, \stackrel{*}{u})\right] d t \\
& +\rho_{0}\|\theta(t, \stackrel{*}{x}, \stackrel{*}{u})\|^{2} \geqq 0,
\end{aligned}
$$

which in view of the given hypothesis implies that

$$
b_{0}(\stackrel{*}{x}, \stackrel{*}{u})\left[\int_{a}^{b} *^{T} f(t, \stackrel{*}{x}, \stackrel{*}{x}) d t-\int_{a}^{b} \stackrel{*}{T}^{T} f(t, \stackrel{*}{*}, \stackrel{*}{u}) d t\right]>0 .
$$

As $b_{0}(\stackrel{*}{*}, \stackrel{*}{u})>0$, it follows that

$$
\int_{a}^{b} \stackrel{*}{T}^{T} f(t, \stackrel{*}{*}, \stackrel{*}{x}) d t>\int_{a}^{b} *^{T} f(t, \stackrel{*}{\mathcal{u}}, \stackrel{*}{u})
$$

But this contradicts (78), hence the result.

\section{Some Related Problems}

All the optimality conditions and duality results developed for $(V P)$ in the previous sections can easily be modified for several other classes of variational problems.
5.1. Natural Boundary Value Problems. Omitting the boundary conditions for the fixed end points as was done by Mond and Hanson [2], we obtain

$$
\begin{aligned}
\left(V P_{0}\right) \text { minimize } & \int_{a}^{b} f(t, x, \dot{x}) d t \\
& =\left(\int_{a}^{b} f_{1}(t, x, \dot{x}) d t, \ldots, \int_{a}^{b} f_{p}(t, x, \dot{x}) d t\right)
\end{aligned}
$$$$
\text { subject to } h(t, x, \dot{x}) \leqq 0 t \in I \text {. }
$$

The corresponding Mond-Weir type dual is given by

$$
\begin{aligned}
\left(V D_{0}\right) \text { maximize } & \int_{a}^{b} f(t, u, \dot{u}) d t \\
& =\left(\int_{a}^{b} f_{1}(t, u, \dot{u}) d t, \ldots, \int_{a}^{b} f_{p}(t, u, \dot{u}) d t\right)
\end{aligned}
$$

subject to $\quad \lambda^{\mathrm{T}} f_{x}(t, u, \dot{u})+y(t)^{T} h_{x}(t, u, \dot{u})$

$$
\begin{aligned}
& =\frac{d}{d t}\left(\lambda^{T} f_{\dot{x}}(t, u, \dot{u})+y(t)^{T} h_{\dot{x}}(t, u, \dot{u})\right), \\
& y(t) \geq 0, \quad t \in I, \\
& {\left[y(t)^{T} h_{x}(t, u, \dot{u})\right]_{t=a}=0,} \\
& {\left[y(t)^{T} h_{\dot{x}}(t, u, \dot{u})\right]_{t=b}=0,} \\
& {\left[\lambda^{T} f_{x}(t, u, \dot{u})\right]_{t=a}=0,} \\
& {\left[\lambda^{T} f_{\dot{x}}(t, u, \dot{u})\right]_{t=b}=0,} \\
& \lambda \geqq 0, \quad \lambda^{T} e=1, \\
& e=(1,1, \ldots, 1) \in \mathbb{R}^{p} .
\end{aligned}
$$

If the problems $\left(V P_{0}\right)$ and $\left(V D_{0}\right)$ are independent of $t$, then these problems essentially reduce to the static case $(V \bar{P})$ and $(V \bar{D})$ of multiobjective nonlinear programs:

$$
\begin{aligned}
(V \bar{P}) \text { minimize } & f(x)=\left(f_{1}(x), f_{2}(x), \ldots, f_{p}(x)\right) \\
\text { subject to } & h(x) \leq 0, \\
(V \bar{D}) \text { maximize } & f(u)=\left(f_{1}(u), f_{2}(u), \ldots, f_{p}(u)\right), \\
\text { subject to } & \lambda^{T} f_{x}+y(t)^{T} h_{x}=0, \\
& y \geqq 0, \quad \lambda \geqq 0, \quad \lambda^{T} e=1, \\
& \quad e=(1,1, \ldots, 1) \in \mathbb{R}^{p} .
\end{aligned}
$$

\section{Acknowledgments}

The authors wish to thank the referees and the Editor for their valuable suggestions which improved the presentation of the paper. 


\section{References}

[1] M. A. Hanson, "Bounds for functionally convex optimal control problems," Journal of Mathematical Analysis and Applications, vol. 8, no. 1, pp. 84-89, 1964.

[2] B. Mond and M. A. Hanson, "Duality for variational problems," Journal of Mathematical Analysis and Applications, vol. 18, no. 2, pp. 355-364, 1967.

[3] C. R. Bector and I. Husain, "Duality for multiobjective variational problems," Journal of Mathematical Analysis and Applications, vol. 166, no. 1, pp. 214-229, 1992.

[4] C. Nahak and S. Nanda, "Duality for multiobjective variational problems with invexity," Optimization, vol. 36, no. 3, pp. 235-248, 1996.

[5] D. Bhatia and A. Mehra, "Optimality conditions and duality for multiobjective variational problems with generalized Binvexity," Journal of Mathematical Analysis and Applications, vol. 234, no. 2, pp. 341-360, 1999.

[6] B. Mond, S. Chandra, and I. Husain, "Duality for variational problems with invexity," Journal of Mathematical Analysis and Applications, vol. 134, no. 2, pp. 322-328, 1988.

[7] G. J. Zalmai, "Optimality conditions and duality models for a class of nonsmooth constrained fractional variational problems," Optimization, vol. 30, pp. 15-51, 1994.

[8] J. C. Liu, "Optimality and duality for generalized fractional variational problems involving generalized $(\mathrm{F}, \rho)$-convex functions," Optimization, vol. 37, no. 4, pp. 369-383, 1996.

[9] M. A. Hanson and B. Mond, "Necessary and sufficient conditions in constrained optimization," Mathematical Programming, vol. 37, no. 1, pp. 51-58, 1987.

[10] N. G. Rueda and M. A. Hanson, "Optimality criteria in mathematical programming involving generalized invexity," Journal of Mathematical Analysis and Applications, vol. 130, no. 2, pp. 375-385, 1988.

[11] C. Nahak and S. Nanda, "Multiobjective duality with $\rho-$ $(\eta, \theta)$-invexity," Journal of Applied Mathematics and Stochastic Analysis, vol. 2005, no. 2, pp. 175-180, 2005.

[12] V. Chankong and V. V. Haimes, Multiobjective Decision Making: Theory and Methodology, North-Holland, New York, NY, USA, 1983.

[13] A. M. Geoffrion, "Proper efficiency and the theory of vector maximization," Journal of Mathematical Analysis and Applications, vol. 22, no. 3, pp. 618-630, 1968. 

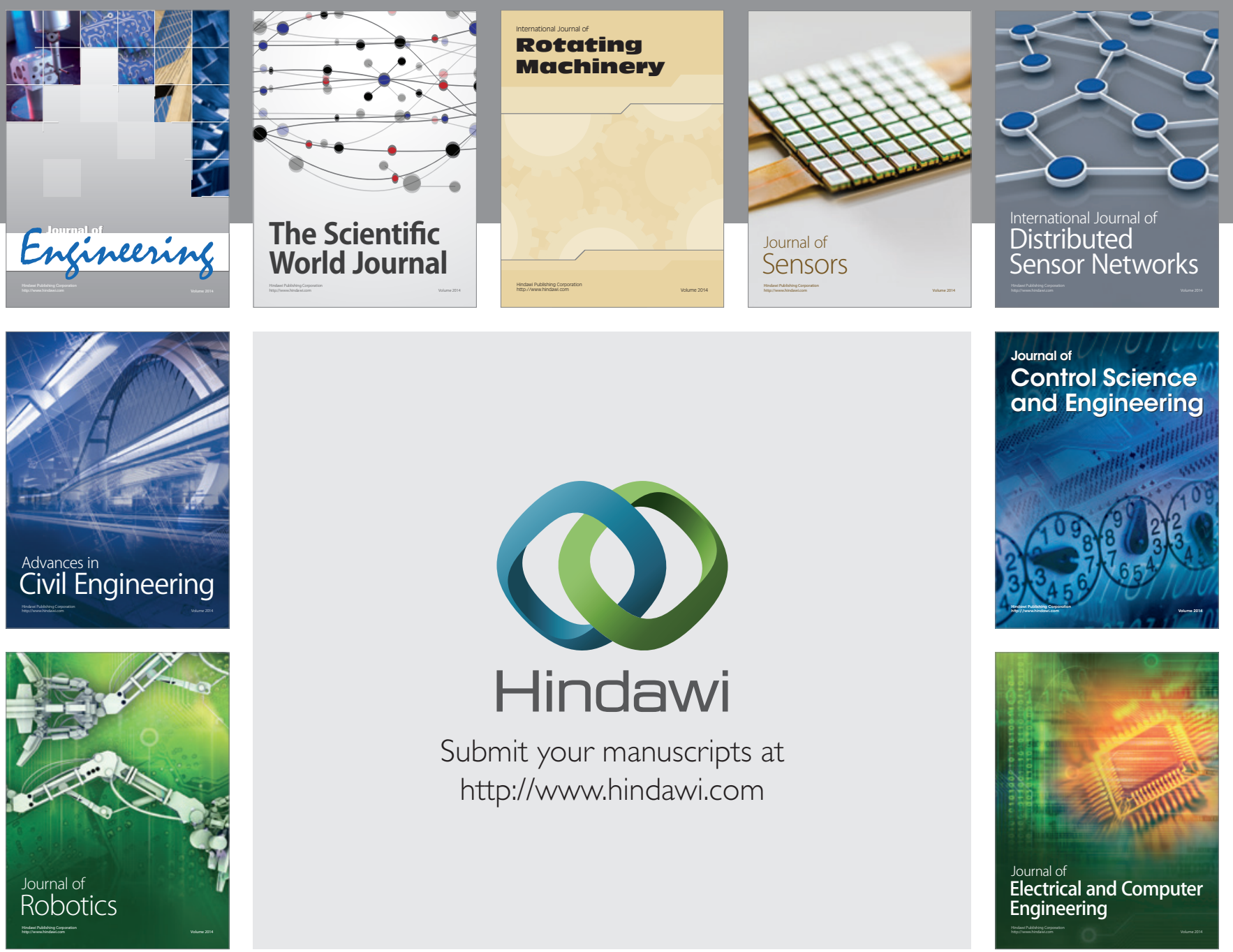

Submit your manuscripts at

http://www.hindawi.com
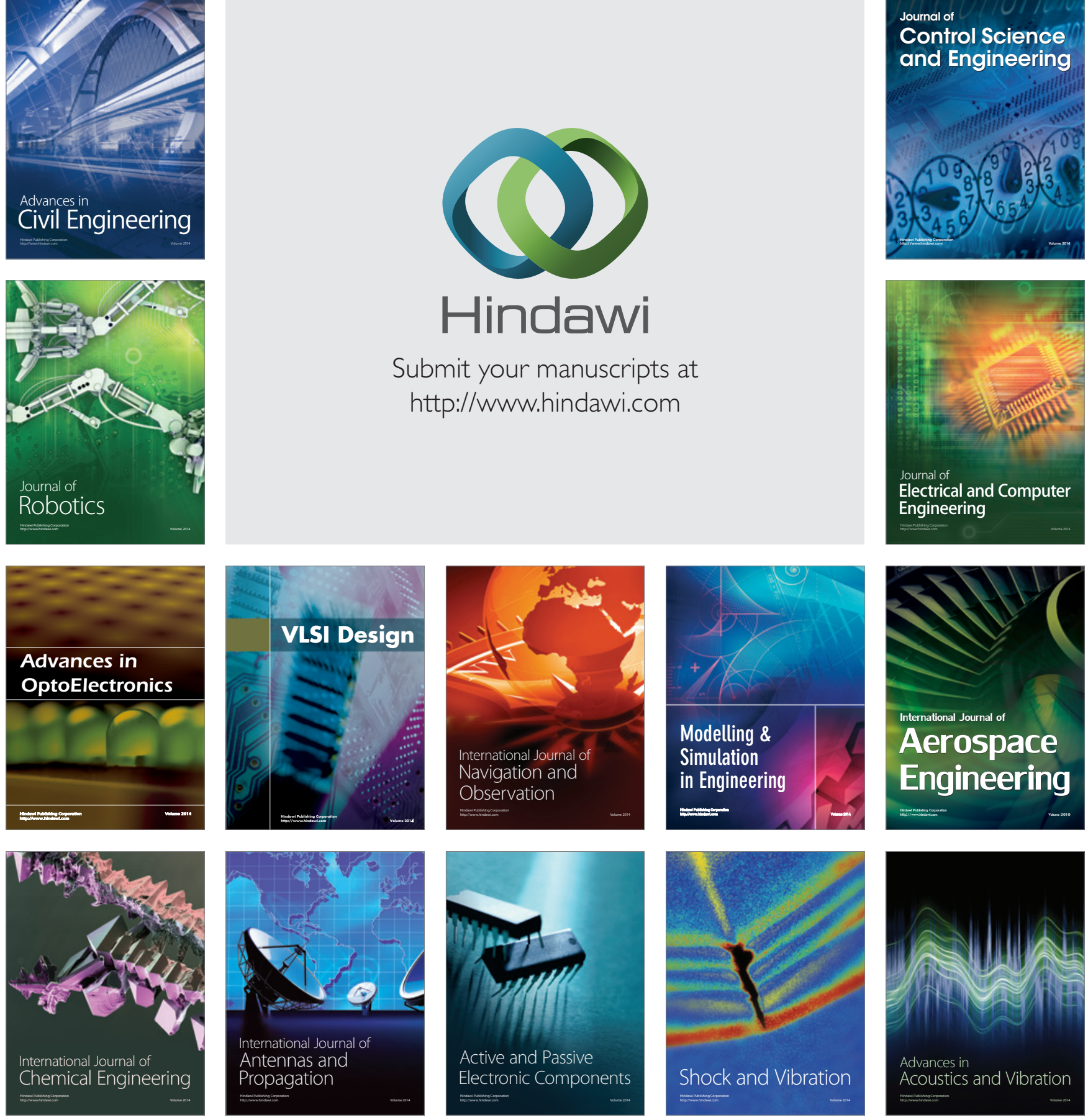